\title{
TMS320C6713 DSP Kit based Hardware Implementation for the Microphone Array Beamforming System
}

\author{
Mahmod A. Al Zubaidy \\ Ninevah University \\ Mosul, Iraq
}

\author{
Shatha M. Ali \\ School of Electronics Engineering \\ Mosul, Iraq
}

\begin{abstract}
It is so difficult to get a pure single acoustic signal, where acoustic signals often suffer from noise and interferences. Also in some cases there are several sources of acoustic signals in the zone, one of them is desirable and the others are required to be cancelled. The Microphone Array Beamforming System (MABFS) is one of the best solutions for the purpose of extracting one signal from several signals by directing the main lobe of the microphone array towards the desired signal and placing the other signals in the nulls of this array. In this paper three types of MABFS were implemented based on TMS320C6713 DSP KIT, these systems are uniform phase and amplitude MABFS using two microphone, uniform phase and amplitude MABFS using four microphones, and nonuniform phase and amplitude MABFS using four microphones. All the implemented systems were tested with sine wave signals and then with recorded speech signals, the results of these systems were compared with each other and with the MATLAB SIMULINK results that worked in past.
\end{abstract}

\section{Keywords}

Noise and interference cancellation, uniform and non-uniform amplitude and phase, adaptive Filtering, beamforming,

Microphone array, acoustic digital signal processing, TMS320C6713 KDS KIT.

\section{INTRODUCTION}

Beamforming is a signal processing technique used in many applications in the communication system. [1] This is achieved by combining array element in such a way that signals at particular angles experience constructive interference while others experience destructive interference. This technique achieves spatial selectivity and can be applied to radio or sound waves [2]. Usually, this system used to reduce the signal-ofinterest at the output of a microphone array by means of best (e.g., least-squares), it represents a spatial filter which can extract a signal from a specific direction and reduce the signals from other directions [3].

In general, there are many types of beamforming system, the generalized sidelobe canceller (GSC) is a largely used to separate interference and noise from the desired signal which illustrated in fig1, the GSC consists of three system processing units:-

1-The fixed beamformer (FBF) which is designed to get desired speech signal.

2-The blocking matrix (BM) which represents the manner of spatial rejection filter supposed to block the speech signal parts in the microphone signals. It rejects the desired signal and passes interference.
3- The interference references at its output drive a multichannel adaptive interference canceller (AIC) whose coefficients are adapted to suppress the remaining noise in the FBF output.

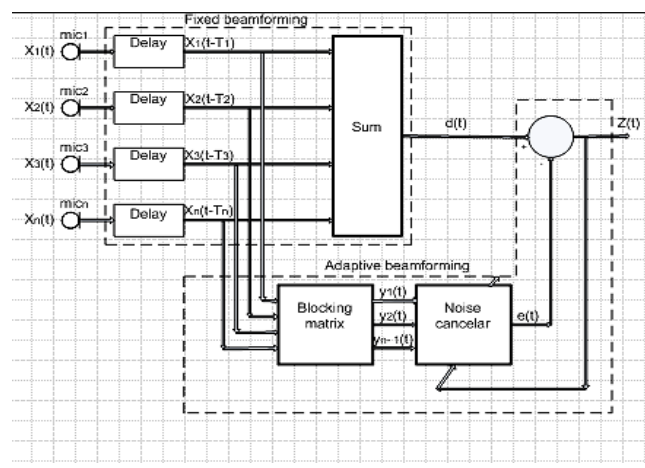

Fig.1: Units of Generalized Side-lobe canceller system

$d(t)$ is the output of fixed beamforming path

$y_{n}(t)$ is the output of blocking matrix

$e(t)$ is the output of adaptive interference canceller

$z(t)$ is the output of adaptive beamforming system

\section{THEORY OF (MABFS)}

When designing microphone array system we need to a number of microphones equal $\mathrm{N}$. The input of each microphone sound signals equal I, each signal of them have specific arrival angle. As shown in fig.2 [4]. Each microphone in the array will thus receive all of these signals $s i$, but it received each signal with a delay depends on the distance between the sources and the microphones. If the distance from microphone $\mathrm{m}_{1}$ to any microphone $\mathrm{m}_{n}$ is $\mathrm{d}_{n}$. The time delay can be calculated [4].

Time delay $(\tau)=\frac{d n \cos \theta i}{v}$

$\mathrm{v}$ is the velocity of sound $(343 \mathrm{~m} / \mathrm{s}), \theta \mathrm{i}$ is the incident angle.
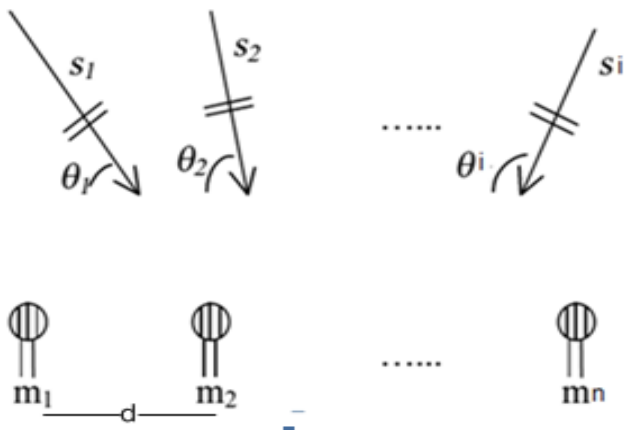

Fig.2 Microphone array layout 
The signal arrival to each microphone $X_{n}(t)$

$$
\begin{aligned}
& X n(t)=s i(t-\tau) \\
& \boldsymbol{X n}(\boldsymbol{t})=\boldsymbol{s i}(\boldsymbol{t}) \boldsymbol{e}^{-\boldsymbol{j} \boldsymbol{\omega} \boldsymbol{\tau}} \\
& \omega=2 \pi f c
\end{aligned}
$$

$\mathrm{f}_{\mathrm{c}}$ is the cutoff frequency

the incoming signals are a combination of noise and $\boldsymbol{I}$ signals so it can be calculated as:

$$
X n(t)=1 / N \sum_{i=1}^{I} \operatorname{Amsi}(t) e^{-j 2 \pi f c d n c o s \theta i / v}
$$

$A_{m}$ is the amplitude of each microphone.

For uniform microphone array, the delay $\left(T_{n}\right)$ can calculate as:

$$
\operatorname{Tn}=\frac{d n \cos \beta}{v}
$$

Where $\beta$ is the direction of the desired signal.

While (LMS) algorithm used to calculate the amplitude and phased parameters in non-uniform beamforming. While the output of (FBF) can get by summation of the all microphones signals. All these equations were implemented and all variables were calculated and applied through the use of a program MATLAB-SIMULATION in [4].

The output of the first part of the ABF (blocking matrix) (BM) represents the difference between successive output microphone signal [4]. The second parts of the adaptive path (adaptive input canceller) (AIC) contain an LMS algorithm adaptive filter which has variable parameters calculated depending on the output of fixed beamforming signal and subtracts components (the output of BM) [5].

\section{HARDWARE SYSTEM IMPLEMENTATION USING DSP KIT}

DSP processors are most commonly used in real-time signal processing [6]. For real-time processing, external factors must be considered, while when the signal is processed in a nonreal-time processing, there are no time limits. For a system that is not real time, data can be recorded on the computer and be using known algorithms used in signal processing. DSP based systems are less susceptible to environmental conditions. DSP Processors have microprocessor characteristics [7]. They are user-friendly, flexible and economical [8]. The perfect reconstruction code has been successfully executed on TMS320C6713 several DSP. The use of DSP kit in real-time signal processing system requires the following assistant's equipment and programs:

MATLAB-SIMULINK model for the system, code composer studio program (CCS), Integrated Development Environment (IDE) link between MATLAB and code composer studio,

Embedded $\mathrm{ABF}$ is comprised of components that shown in fig.3.

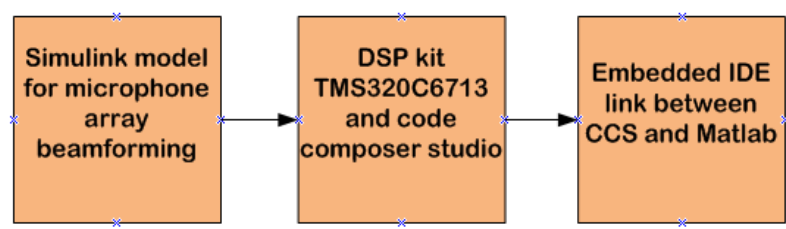

Fig.3: The requirements for implementation the real time signal processing system when using DSP kit

\section{HARDWARE IMPLEMENTATION FOR THE MABFS.}

In this work three types of MABFS will be implemented, the first one is the uniform amplitude and phase MABFS using two microphones and the other are uniform and non-uniform amplitude and phase MABFS using four microphones.

\subsection{UNIFORM MABFS USING TWO MICROPHONES}

The implementation of the uniform microphone array beamforming system using two microphones system needs one TMS320C6713 KDS KIT, three PC, and oscilloscope, fig.4 shows the block diagram of this implemented system.

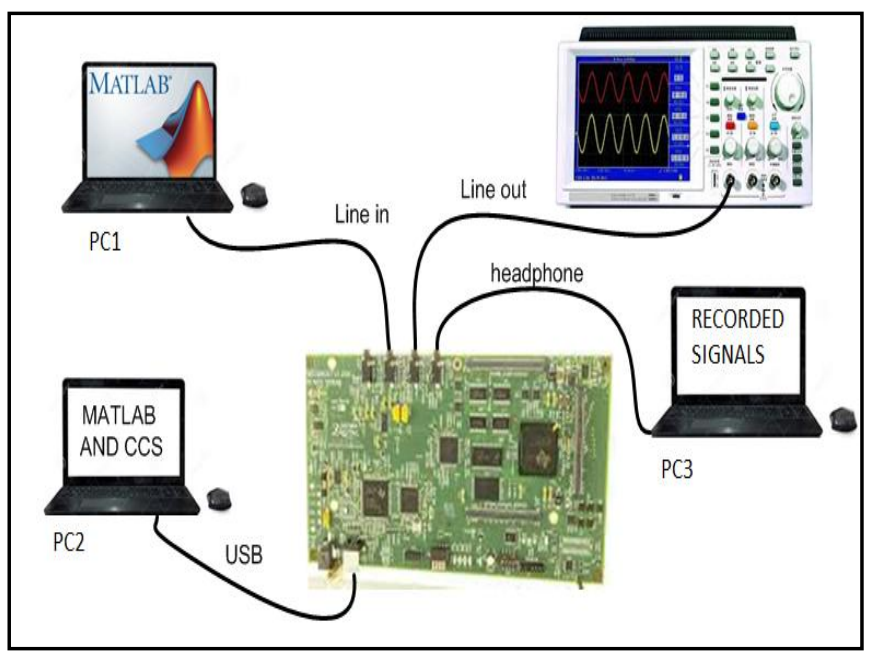

Fig.4: TMS320C6713 KDS KIT based hardware implementation system of the MABFS using two microphones

PC1 that connect to the KDS KIT at stereo line connector are representation the outputs of the two microphones signals. These signals generated in the PC1 and send to the KDS KIT through the audio outputs of the PC.

Two multimedia file blocks are used to generate the two microphones signals; in each block the signal output of one microphone is recorded. Then used matrix concatenate block to synchronization between the two signals, the MATLABSIMULINK circuit that used to generate the two microphone signals are shown in fig.5.

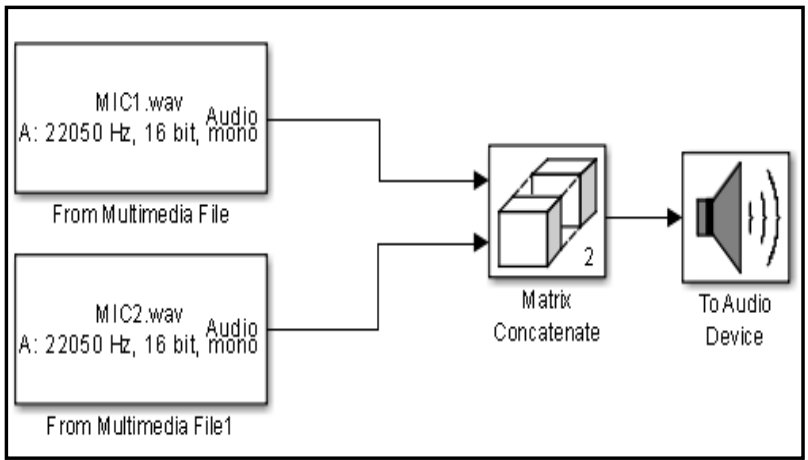

Fig.5: MATLAB-SIMULINK circuit that used to generate the two microphone signals (in Pc1)

PC2 represent the host computer for the KDS KIT, in this computer, the KDS KIT codes are generated using MATLAB-SIMULINK program and CCS program. This 
generated code is loaded to the KDS KIT by USB cable that connects to the PC and the KIT fig. 6 shown the MATLABSIMULINK circuit.

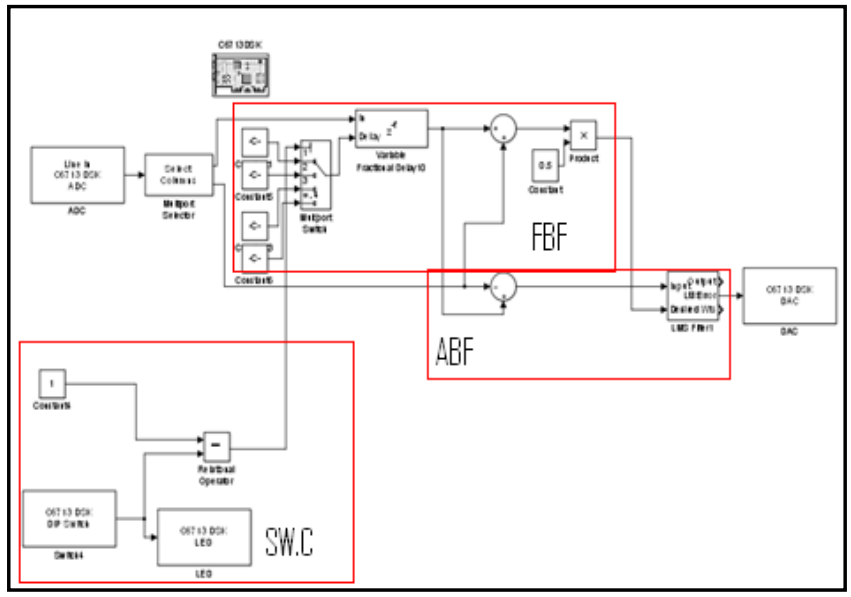

Fig.6: MATLAB SIMULINK circuit for the MABFS using 2MICs 4sources based on TMS 320c6713kds KIT

The purpose of using PC3 that connect to KDS KIT from headphone connector is to record the output signal for the implemented system. The first channels of the oscilloscope are connected to the Line out connector of the KDS KIT to show the output signal, while the other channels of this oscilloscope are connected to the outputs of the PC1 in parallel to the inputs point of the KDS KIT to show the inputs signals.

\subsection{UNIFORM MABFS USING FOUR MICROPHONES.}

When using 4 microphones in implementation of the MABFS there are a need of two TMS320C6713 KDS KITs, four PC and oscilloscope, fig.7 shown the block diagram of the hardwareimplementation of this system.

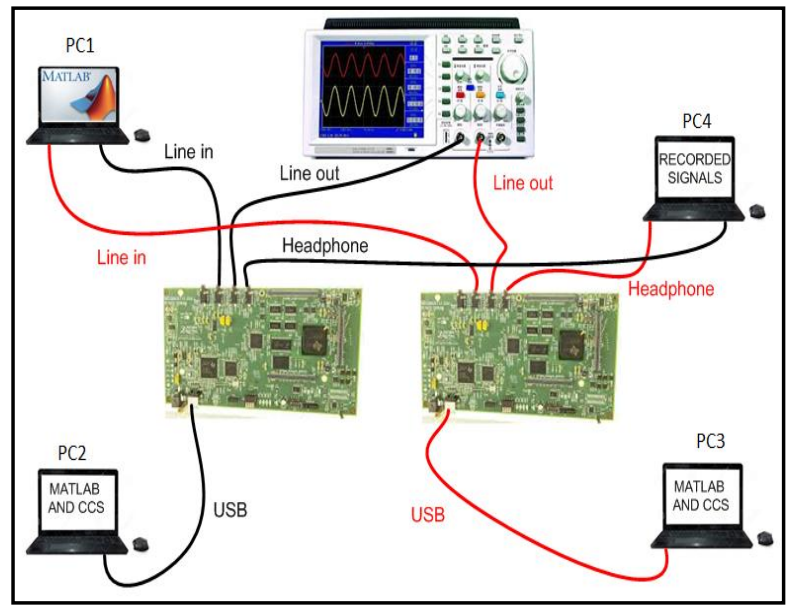

Fig.7: TMS320C6713 KDS KIT based hardware implementation system of the MABFS using four MICs

PC2 which connected to the KDS KIT 1 at stereo line connector represents the outputs of the two microphones signals. These signals generated in the $\mathrm{PC} 1$ and send to the KDS KIT1 through the audio outputs of the PC1. Also, PC3 are connected to the KDS KIT 2 at stereo line connector are represent the outputs of the other two microphones signals. These signals generated in the PC1 and send to the KDS KIT2 through the USB audio device connector fig.8, 9 shown the MATLAB-SIMULINK circuit.

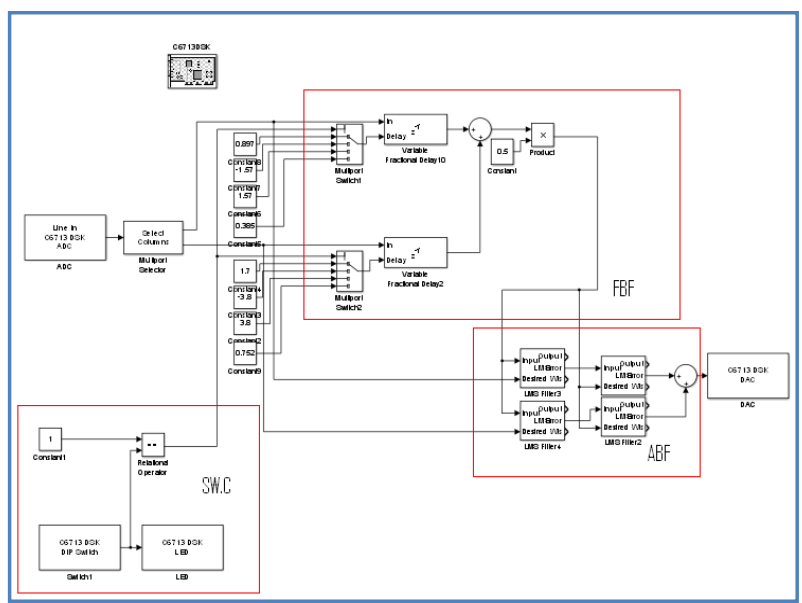

Fig.8: MATLAB SIMULINK circuit for the MABFS using 4MICs 4sources based on TMS 320c6713kds KIT part 1 (pc2)

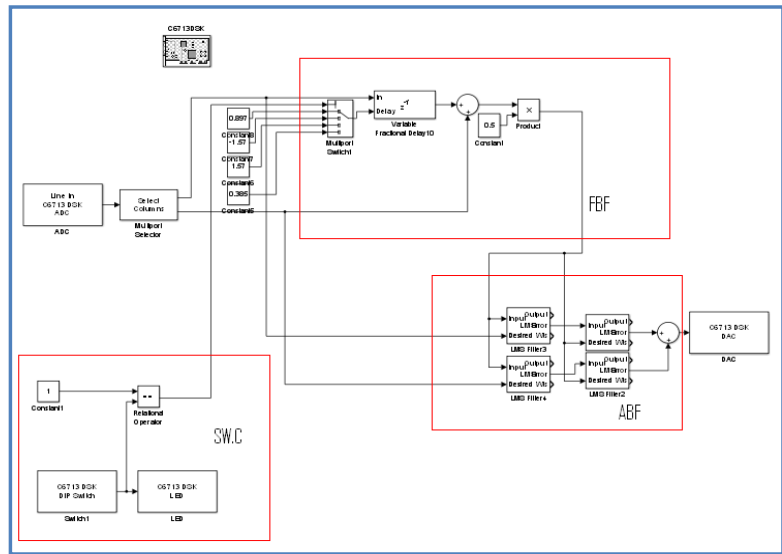

Fig.9: MATLAB SIMULINK circuit for the MABFS using 4MICs 4sources based on TMS 320c6713kds KIT part 2 (pc3)

Four multimedia file blocks are used to generate the four microphones signals; in each block the signal output of one microphone is recorded. Then used two matrixes concatenate blocks to synchronization between the four signals; each two input signals connected to one matrix concatenate block, the MATLAB-SIMULINK circuit that used to generate the four microphone signals are shown in fig. 10



Fig.10: MATLAB-SIMULINK circuit that used to generate the four microphone signals (in PCI) 
The purpose of using PC4 that connect to KDS KIT from headphone connector is to record the output signal for the implemented system.

PC2 represent the host computer for the KDS KIT1, in this computer, the KDS KIT1 codes are generated using MATLAB-SIMULINK program and CCS program. This generated code is loaded to the KDS KIT1 by USB cable that connects to the PC and the KIT1. While PC3 represents the host computer for the KDS KIT2, in this computer the KDS KIT2 codes are generated using MATLAB-SIMULINK program and CCS program. This generated code is loaded to the KDS KIT2 by USB cable that connects to the PC and the KIT2. Line out of KIT1 connector connect to an oscilloscope at channel1 while Line out of KIT2 connector connects to an oscilloscope at channel 2 to show the signals by using adding mode.

\subsection{NONUNIFORM PHASE AND AMPLITUDE MABFS USING FOUR MICROPHONES}

In order to enhanced signal to interference ratio, nonuniform amplitude and phase microphone array beamforming are used. the way to construct this system has been done by calculation the parameter of the phase and amplitude by using LMS algorithm.this prammeter added to system at fig. 8 and 9 then apply at TMS320C6713KDS KIT 1 and TMS320c6713kds KIT 2.

\section{RESULTS}

For the all implemented systems $(\mathrm{N}=2$ or $\mathrm{N}=4)$ uniform and non-uniform phase and amplitude, the microphones with an interelement distance of $\mathrm{d}=0.057 \mathrm{~m}$ placed in a simulated reverberant enclosure of the size $(5 \mathrm{~m}) \times(5 \mathrm{~m})$ was used. The speech sources were placed with angles of $\theta 1=10$ degree, $\theta 2=$ 60degree, $\theta 3=120$ and $\theta 4=135$. And the $\mathrm{fc}$ of the speech signals are $\mathrm{S} 1=700 \mathrm{~Hz}, \mathrm{~S} 2=2000 \mathrm{~Hz}, \mathrm{~S} 3=1150 \mathrm{~Hz}, \mathrm{~S} 1=3000 \mathrm{~Hz}$. These four signals are shown in fig. 11 .

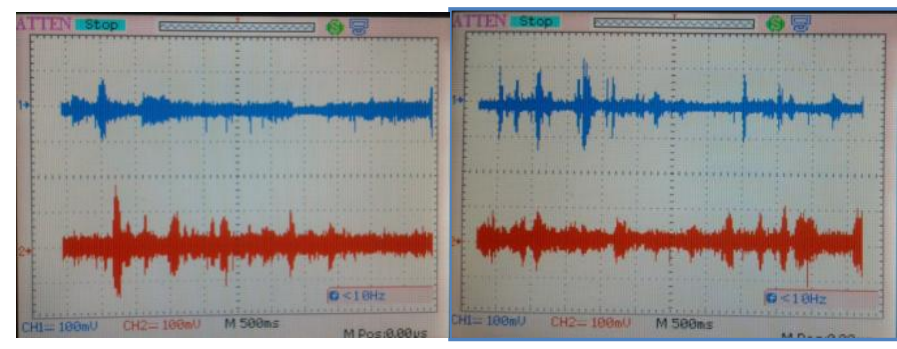

Fig.11: four speech input signals

\subsection{HARDWARE IMPLEMENTATION RESULTS FOR THE UNIFORM MABFS USING TWO MICROPHONES}

Fig.12 shows the input speech signals at each two microphones. As well as the first microphone assumed as a reference, his input will be the summation of the four signals without delay, while the input of the other microphones must be the summation of the four signal but each signal suffer from delay that calculated by the four calculation blocks by using matlab simulink programm.

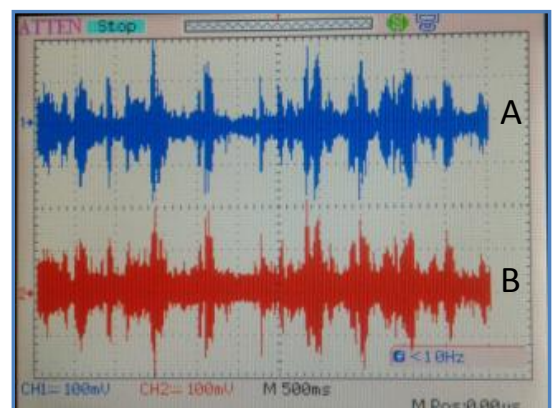

Fig.12: The input speech signals to each microphone in case (2MICs, 4 sources) A (ref.MIC.) B MIC2 signals

Fig.13 shown that the output of system when control the beam through the SW.C and change the time delay, This output is the desired signal in addition to the remaining interference that has not been deleted from the system.

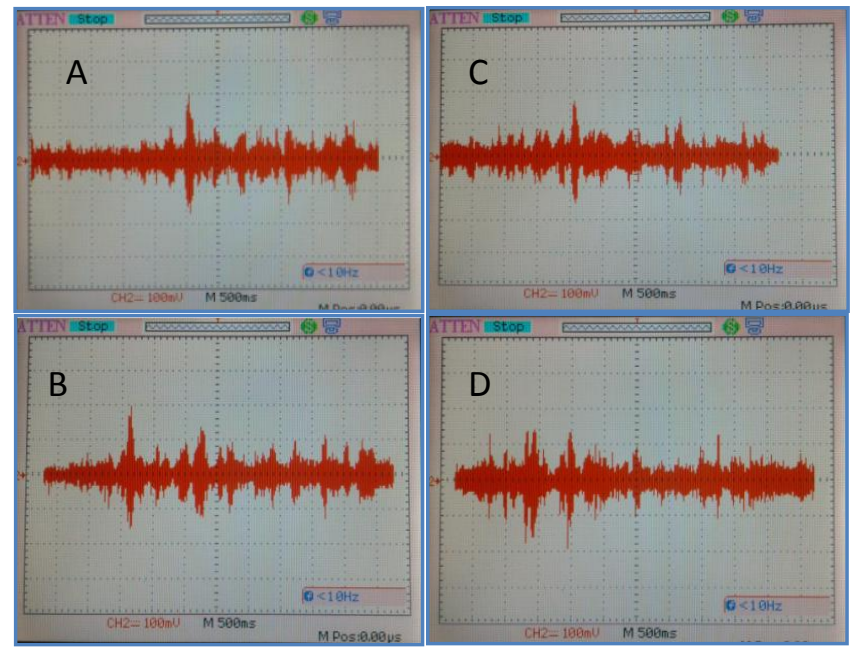

Fig.13: tow MICs 4sources using tms320c6713kds KIT output system tested by four speech signals

A output ABF when the beam direction the signal at theta1

$B$ output $A B F$ when the beam direction the signal at theta2

C output ABF when the beam direction the signal at theta3

D output ABF when the beam direction the signal at theta4

\subsection{HARDWARE IMPLEMENTATION RESULTS FOR THE UNIFORM MABFS USING FOUR MICROPHONES}

Fig.14 shows the input speech signals at each four microphones.

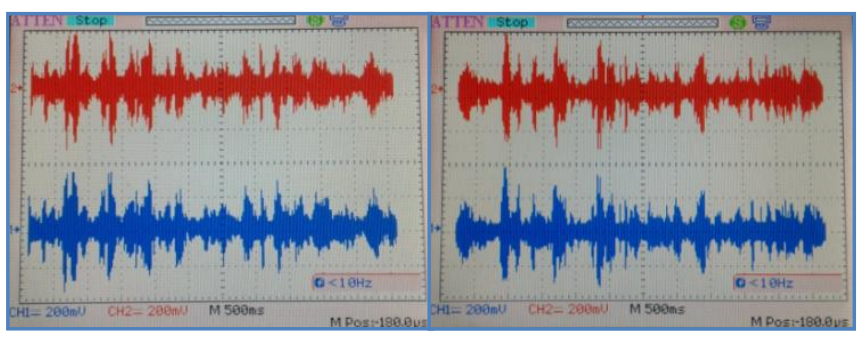

Fig.14: The input speech signals to each microphone in case (4mics , 4 sources) 
From fig.15 channel 1 representation the output of KIT1 channel 2 representation the output of KIT2, the output of the system represents the desired signal and the remaining of three interference signals.
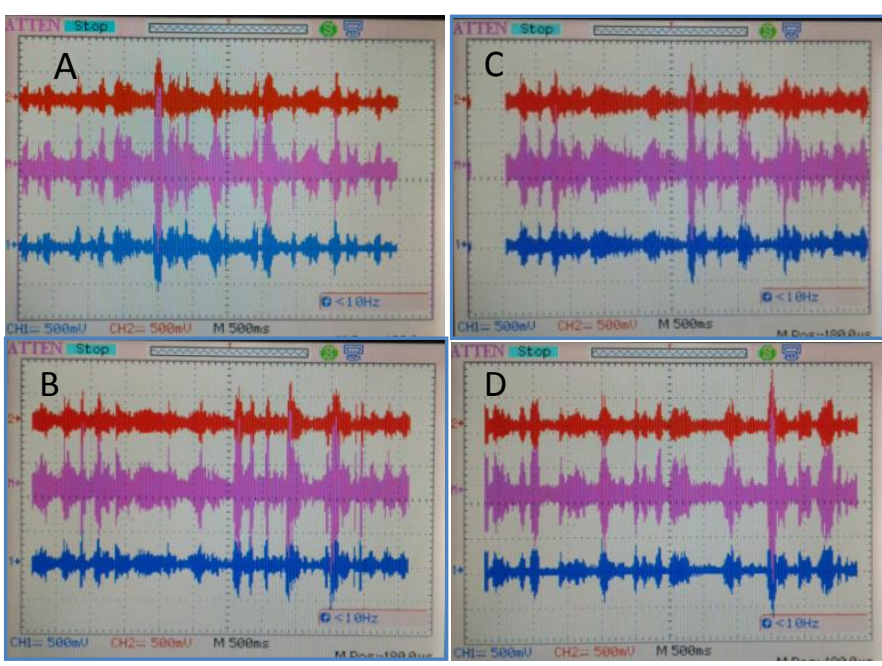

Fig.15: 4MICs 4sources using tms320c6713kds KIT output system tested by four speech signals

A output ABF when the beam direction the signal at theta1

$B$ output ABF when the beam direction the signal at theta2

$C$ output $A B F$ when the beam direction the signal at theta 3

$D$ output ABF when the beam direction the signal at theta4

\subsection{HARDWARE IMPLEMENTATION RESULTS FOR THE NON-UNIFORM MABFS USING FOUR MICROPHONE}

The inputs of this system are shown in fig.14.the outputs of system are shown in fig.16. For speech signals and when implementation non-uniform amplitude and phase the interference signals in output system are fewer Compared to the output of another system.
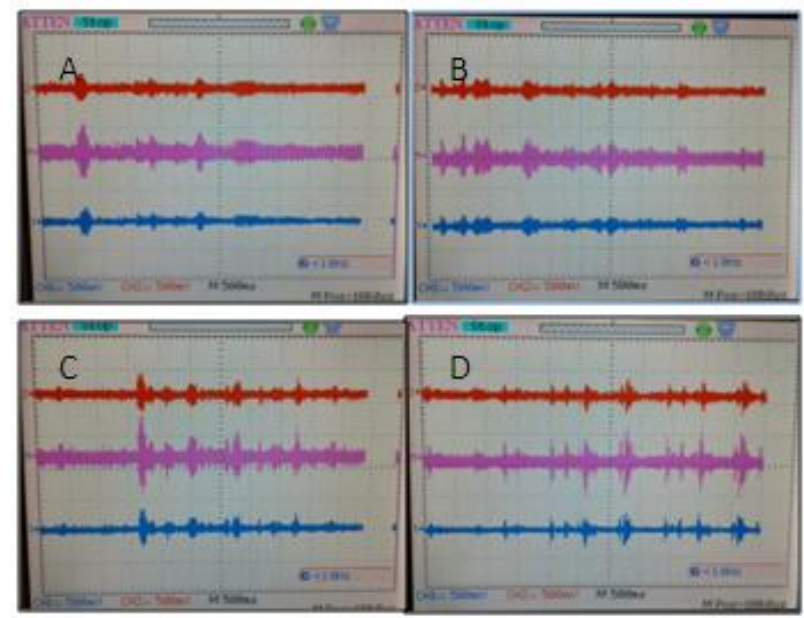

Fig.16: 4MICs 4sources using TMS320C6713KDS KIT output system tested by four speech signals (non-uniform phase and amplitude beamforming system)

A output ABF when the beam direction the signal at theta1 $B$ output $A B F$ when the beam direction the signal at theta2

C output ABF when the beam direction the signal at theta 3 $D$ output ABF when the beam direction the signal at theta4

\section{CONCLUSION}

From the previous work it can be concluded the following:-

1 - In general the microphone array beamforming system is an efficient technique to cancel three unwanted interfacing signals from four signals, this process done by steering the nulls of the beamforming array toward the unwanted signals.

2 - The use of the TMS320C6713 KDS KIT are so suitable for real time acoustic digital signal processing, and so flexible for implementation these systems.

3 - The results of the three hardware implemented systems indicates that the uniform MABFS using four microphones enhanced the signal to noise ratio by about $3 \mathrm{~dB}$ above the $\mathrm{S} / \mathrm{N}$ ratio for the uniform MABFS using two microphones, while the non-uniform MABFS using four microphone enhanced the $\mathrm{S} / \mathrm{N}$ ratio by about $5 \mathrm{~dB}$ above the $\mathrm{S} / \mathrm{N}$ ratio for the uniform MABFS using four microphone, and this clearly shown in fig.17.

4 - The hardware results show so small differences of the S/N ratio from the MATLAB simulation results shown in fig.18 [9].

5 - The future work can be thought by implemented the MABFS using more than four microphones (eight or more) and using other technique like FPGA.

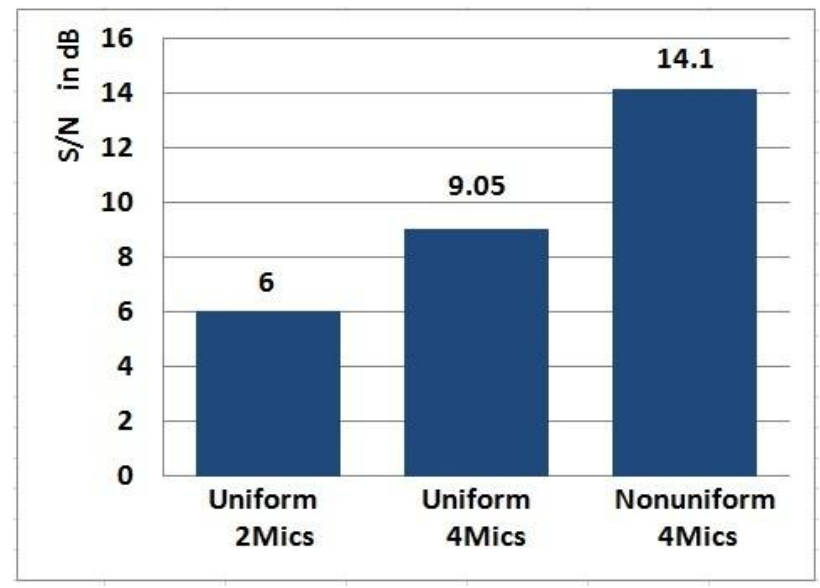

Fig.17: The S/N for the MABFS

(Hardware implementation results)

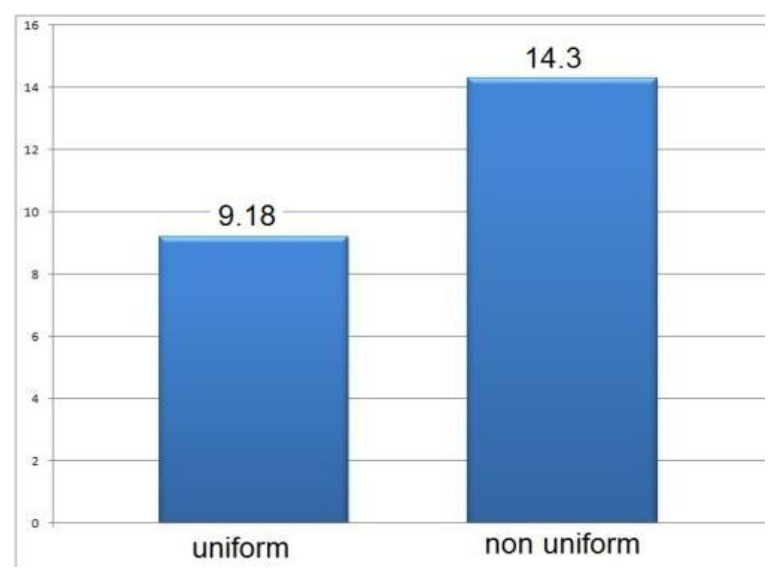

Fig.18: The $S / N$ in $d B$ of the MABFS using 4 microphone (MATLAB simulation results) 


\section{REFERENCES}

[1] S. V. Vaseghi, "Advanced Digital Signal Processing and Noise Reduction", Second Edition, John Wiley \& Sons Ltd, 2000.

[2] Barry Van Veen and Kevin M. Buckley, "Beamforming: a versatile approach to spatial filtering" IEEE Signal Processing Magazine, vol. 5, pp. 4-24, 1988.

[3] H. Levitt, "Noise reduction in hearing aids: An overview," Journal of Rehabilitation Research and Development, vol. 38, no. 1, Jan. 2001.

[4] David K. Campbell, "Adaptive Beamforming Using a Microphone Array for Hands-Free Telephony", MS.C Thesis, Blacksburg, Virginia in 1999.

[5] Hoshuyama,AkihikoSugiyama,And Akihiro Hirano. Ieice Tran. Fundamentals, "A Robust Adaptive Beamforming with a Blocking Matrix Using Coefficient Constrained Adaptive Filter" Osamu VOL.82-A,NO.4 APRIL 1999.

[6] N. kehtarnavaz, "Real-time digital signal processing based on the TMS320C6000", ELSEVIER, 2005.

[7] R. Chassaing, "Digital Signal processing and applications with the C6713 and C6416 DSK", A John Wiley and Sons, Inc., Publications, 2005.

[8] (2010, May.) [Online]. Available: Texas instruments DSP developer's village. World Wide Web, www.dspvillage.ti.com/

[9] Mahmod A. alzubaidy \& shatha M. Ali, "Study and Evaluation of The Uniform and Non-uniform Beam forming Systems for Reduction the Noise and Interference", International Journal of Engineering and Innovative Technology (IJEIT), Volume 7, Issue 2, August

2017. 